\title{
The Acute Effects of Different Forms of Suspension Push-Ups on Oxygen Consumption, Salivary Testosterone and Cortisol and Isometric Strength
}

\author{
by \\ David Bellar ${ }^{1}$, Cory Etheredge ${ }^{1}$, Lawrence W. Judge ${ }^{2}$
}

Suspension exercise systems are being used in strength and conditioning facilities, fitness centers, rehabilitation centers and home gyms. Though some evidence exists regarding the impact of training with these systems, more work is needed for a better understanding. The purpose of the present investigation was to examine the acute effects of an exercise session with 2 (hands only) and 4 straps (hands and feet) in the push-up exercise compared to a work-matched bench press exercise session. The participants for this repeated measures, cross-over investigation were 18 healthy college-aged males (age: $24.8 \pm 3.5$ yrs, body mass: $81.8 \pm 7.8 \mathrm{~kg}$, body height: $178.9 \pm 4.5 \mathrm{~cm}$ ). The conditions were 6 sets of 10 repetitions of suspension push-ups using two straps (DUAL) for the hands, fours straps (QUAD) for hands and feet and a traditional bench press exercise matched to the average resistance during the suspension push-up. The participants performed all repetitions at a controlled cadence. Expired gases, and heart rate were monitored continuously during the exercise session. Pre and post exercise saliva samples were collected to quantify changes in testosterone and cortisol. Upper body isometric strength tests (UBIST) were performed (Post, $1 \mathrm{hr}$, $24 \mathrm{hr}, 48 \mathrm{hr}$ ) to evaluate changes in force production during recovery. Data analysis via repeated measures ANOVA revealed a significant trend for increased oxygen consumption in the QUAD condition compared to the bench press $(p=$ 0.019). Additionally, both suspension conditions resulted in a reduced respiratory exchange ratio as compared to the bench press $(p<0.05)$. A significant main effect was noted for time in all conditions regarding isometric strength $(p<$ 0.001), but no differences between conditions were revealed. Testosterone and cortisol responses did not differ between conditions. Based upon these data, it appears that when matched for work, suspension exercise results in equivalent reductions in muscle force, but greater oxygen consumption compared to isotonic exercise.

Key words: unstable exercise, suspension, exercise metabolism, testosterone, cortisol.

\section{Introduction}

There are many exercise devices that are designed to increase the difficulty of overcoming the weight of the body or a barbell due to instability. Suspension exercise trainers offer instability during exercises that mimic traditional calisthenics. These devices, and exercises in general that involve an element of instability, have been shown to elicit similar or elevated surface EMG reading compared to traditional resistance exercises (Calatayud et al., 2014; Maszczyk et al., 2016). Byrne et al. (2014) reported that compared to a floor based plank exercise, suspension exercise resulted in higher activation in the lumbopelvic muscles. This echoed the results reported by Snarr and Esco (2014) who indicated that adding multiple instability devices to a plank exercise also elicited greater muscle activation. Mok et al. (2015) offered further evidence that a hip abduction in a plank exercise, performed with suspension, had the strongest potential to active lumbopelvic muscles. In a different exercise mode, the push-up, suspension

1 - School of Kinesiology, University of Louisiana at Lafayette, Lafayette LA USA 70503.

2 - School of Kinesiology, Ball State University, Muncie IN USA 47306. 
exercise has been shown to produce higher levels of activation in the prime movers as compared to the traditional exercise form (Snarr and Esco, 2014). Though a more recent investigation showed that not all prime movers were significantly elevated when comparing suspension and traditional push-ups (Borreani et al., 2015). Further evidence from a study of the inverted row showed an equivalent or reduced signal compared to a non-suspension form of the exercise (Snarr and Esco, 2013). Differences among these results may be due to variation in exercise selection and methodologies.

Only two studies to date have examined cardiovascular and metabolic demands of suspension exercise training (Dudgeon et al., 2015; Snarr et al., 2014). When examining suspension trainers used in high-intensity interval-training (HIIT), it was reported that the exercise elicited moderate heart rates and oxygen consumption (Snarr et al., 2014). In this study the exercise resulted in a mean of $55 \%$ of $\mathrm{VO} 2$ max. It is important to understand oxygen consumption of any training exercise as this information is critical to adjusting the dietary requirements of athletes to ensure that they do not fall into caloric deficit. Additionally, this information is also important for coaches to understand the bioenergetic demands of training. Furthermore, it has been noted that understanding the training responses for testosterone and cortisol can be important for modulating adaptation to resistance type exercise (Beaven et al., 2008). Several recent studies have been successful in monitoring this through the measurement of salivary testosterone and cortisol (Beaven et al., 2008; Klentrou et al., 2016). Currently this information is not available for suspension based resistance exercise in the literature.

Training studies have suggested that the use of suspension trainers can improve throwing performance in women's softball (velocity), handball (maximum throwing velocity) and fitness (1 RM bench press) (Prokopy et al., 2008; Saeterbakken et al., 2011; Snarr et al., 2014; Gołaś et al., 2017). This is not surprising, given that loads from these exercises can approach $80 \%$ of body mass in certain movements if performed at body angles near vertical (Melrose and Dawes, 2015).

One problematic issue with these forms of exercise has been the potential for increased loading of the spine due to the instability of suspension. Simply observing these exercises, where the torso is not supported or controlled through any stable points of contact, can lead to concern. For this reason, research to document the spinal load has been undertaken. It was reported that the use of suspension exercise systems only resulted in a modest increase in the load across the spine (Freeman et al., 2006).

Some differences have been noted with suspension exercise when compared to more traditional resistance training, however, more work needs to be done to understand the full impact of this exercise mode. The purpose of the present investigation was to assess oxygen consumption and salivary testosterone and cortisol responses induced by two different suspension push-up exercise sessions against a bench press exercise session that was matched for work. Endocrine responses were examined as traditional resistance exercise has been shown to cause changes in both testosterone and cortisol levels (Gaviglio et al., 2015). A work-matched protocol was chosen to allow for comparison of exercise metabolisms associated with each condition in the experiment.

\section{Methods}

\section{Procedures}

Eighteen healthy active males gave written informed consent to participate in this study (age: $24.8 \pm 3.5 \mathrm{yrs}$, body mass: $81.8 \pm 7.8 \mathrm{~kg}$, body height: $178.9 \pm 4.5 \mathrm{~cm}$ ). The study was a repeated measures design, in which the participants engaged in all three exercise sessions and follow ups in random order. Upon beginning the study, all participants underwent a familiarization session with data collection procedures and exercise modalities. Following the familiarization session participants waited for one week, and then began the exercise trials. The trials consisted of two modes of suspension exercise and a work matched bench press exercise. For each trial saliva was collected pre and post exercise and there were 24 and $48 \mathrm{hr}$ follow-up visits to investigate recovery of isometric strength. A minimum of 7 days passed between exercise trials. The Institutional Review Board at the University of Louisiana at Lafayette reviewed and approved the experimental procedures for ethical 
considerations and the inclusion of human subjects.

\section{Familiarization Session}

For this investigation each participant underwent a familiarization session. The biacromial width of each subject was assessed and used to set grip width standard at $150 \%$ of this measurement for all remaining movements. The average resistance during a suspended push-up was determined by hanging suspension straps from a Load Star Loadcell (1000 lb capacity, sampling rate of $133 \mathrm{~Hz}$ ) and averaging the readings when the participant stopped at the top and bottom positions of the push-up.

\section{top support weight + bottom support weight}

The participants then practiced an isometric force production test at 90 degrees of elbow flexion, and also practiced the push-up with hands in straps and feet on a platform equal to hand height (DUAL), push-ups with hands and feet simultaneously suspended by straps at equal heights (QUAD) and bench press movements. The weight on the bench press was set to the weight determined to represent the average resistance in a suspended push-up. The cadence was held constant during the practice sets of 10 repetitions using a metronome set to $60 \mathrm{bpm}(1 \mathrm{~s}$ up, $1 \mathrm{~s}$ down).

\section{Exercise Sessions}

Following the familiarization visit the participants returned to perform the DUAL, QUAD and isotonic bench press in randomly assigned order. Prior to the exercise session participants were seated quietly for $10 \mathrm{~min}$ to achieve stable baseline oxygen consumption, after which they were transferred to a Rogue Fitness Cage where the exercise was performed (either suspension or bench press). There were two suspension exercise conditions, DUAL and QUAD. The bench press exercise was performed with a standard $20 \mathrm{~kg}$ barbell, half rack and Olympic weights. During all exercise conditions heart rate and expired gases (breath by breath) were monitored continuously during the baseline and exercise sessions using a ParvoMedic TrueOne 2400 Metabolic Cart (Parvomedic, Sandy UT). This system monitored expired oxygen and carbon dioxide as well as heart rate via a telemetry heart rate strap (Polar Electro Inc, Kempele, Finland). The participants were fitted with a mask and expired gases were collected during the entire exercise sessions, the data file was marked with event tags to indicate the beginning and end of sets. Data were averaged across all set and rest periods. The researcher noted in the collected data the transitions between baseline and exercise periods to allow for later comparison.

These visits consisted of 6 sets of 10 repetitions with $60 \mathrm{~s}$ rest between sets. As in the familiarization visit the participants grip was held constant at $150 \%$ of biacromial width (tape marks on floor to note hand location - suspension exercise, and marks on the bar - bench press) and the cadence was controlled via a metronome at 60 bpm. Before and after each exercise bout, participants provided saliva samples to allow for quantification of testosterone and cortisol hormone levels. The participants also performed the isometric force production test immediately after exercise, as well as 1, 24 and $48 \mathrm{hr}$ after each exercise bout.

\section{Upper Body Isometric Strength Test (UBIST)}

The participants were positioned on three elevated platforms with the chest directly suspended over a load cell anchored into the concrete floor of the lab (iLoad Pro, Loadstar Sensors, Fremont CA). The participants were placed in a push-up style position, with the hands at $150 \%$ of biacromial width, and the elbows at 90 degrees of extension (measured via a goniometer). A thick, non-elastic strap was run over one shoulder and under the opposite shoulder and connected with metal rings to a chain that was tethered to the load cell. This location was selected to reduce the possibility of the strap slipping down the torso during the performance of the test. The participants were positioned so that no slack was apparent in the chain prior to initiation of data capture.

The participants were instructed to keep their backs flat, and push with their hands maximally until told to stop by the researcher. Prior to data capture the load cell was tarred to ensure the weight of the load cell and apparatus were accounted for. The researcher started data collection and verbally instructed the participant to "push as hard as possible". The participants were verbally encouraged during data collection, which was terminated when the force production declined by 50 Newtons from the peak value 
registered. The load cell was set to capture data at the maximum rate $(133 \mathrm{~Hz})$ and the data were exported and analyzed in JMP 11.0 (SAS Institute Inc, Cary NC). Peak force values were isolated from the data and used for subsequent analysis. Previously reported data demonstrated this test to be reliable and highly related to upper body strength (Bellar et al., 2015a, b).

\section{Salivary Testosterone and Cortisol Analysis}

Saliva was collected into $2.0 \mathrm{~mL}$ cryovials via passive drool. The samples were then frozen at $-35^{\circ} \mathrm{C}$ until the completion of data collection. At this time the samples were thawed as centrifuged at $1500 \mathrm{x} \mathrm{g}$ for $10 \mathrm{~min}$ and the supernatant was used for further analysis.

Salivary levels of cortisol and testosterone have been shown to relate to levels in serum (Ahn, 2007; Shirtliff et al., 2011). Saliva samples were stored at $-35^{\circ} \mathrm{C}$ immediately after collection until analysis was undertaken. At that time, samples were allowed to thaw and were spun for $15 \mathrm{~min}$ in a micro centrifuge with the supernatant being drawn off for analysis. Commercially available competitive immunoassay kits (Salimetrics LLC, State College, PA, USA) were used for detection of salivary testosterone (TEST assay sensitivity $1 \mathrm{pg} / \mathrm{ml}$ ), and Cortisol (CORT assay sensitivity $<0.007 \mu \mathrm{g} / \mathrm{dl}$ ). Briefly, samples were introduced to a microtitre plate coated with rabbit antibodies, and competitively bound to the plate with hormone linked to horseradish peroxidase. After binding the plate was washed using an automated plate washer (ELx50, BioTek Winooski VT) and the bound hormone was measured using the reaction of the peroxidase with 3,3'5,5'-Tetramethylbenzidine (TMB). Samples were analyzed via colormetric detection (ELx 808, BioTek Winooski VT) in duplicate and read at $450 \mathrm{~nm}$ (with $630 \mathrm{~nm}$ correction). Interassay and intra-assay coefficients of variation were less than $6 \%$.

\section{Statistical Analysis}

Data were first checked for normality via the Shapiro-Wilk test and then analyzed via repeated measures ANOVA for differences by the exercise type. Post-hoc analysis was undertaken with Tukey-Kramer HSD tests. Statistical significance was set a priori at alpha $>0.05$. All statistical analysis was conducted with JMP Pro 11.0 software (SAS Institute Inc, Cary NC).

\section{Results}

\section{Analysis of Normality}

Data were normally distributed $(p>0.05)$ except for concentrations of testosterone and cortisol. These data were log transformed prior to being analyzed via repeated measures ANOVA.

\section{Results of Heart Rate}

Repeated measures ANOVA (Exercise type*Average HR) did not reveal a significant main effect for the exercise type $(\mathrm{F}=1.03, p=$ 0.374) (Table 1).

\section{Analysis of Expired Gases}

Repeated measures ANOVA (Exercise type ${ }^{*}$ Average $\mathrm{O}_{2}$ consumption) revealed a significant main effect for the exercise type $(\mathrm{F}=$ 4.07, $p=0.023$ ). Pairwise comparisons with the Tukey-Kramer HSD tests revealed that the Quad and Bench press resulted in significantly higher average oxygen consumption over the 6 sets of exercise $(p=0.019)$ (Table 1). Similar analysis did not reveal a significant difference in $\mathrm{VCO}_{2}$ between conditions $(\mathrm{F}=1.84, \mathrm{p}=0.169)$.

Repeated measures ANOVA (Exercise type*Respiratory Exchange Ratio) revealed a significant main effect for the exercise type $(\mathrm{F}=$ 15.04, $p<0.001$ ). Pairwise comparisons with Tukey-Kramer HSD tests revealed that the Quad and Dual exercise resulted in significantly lower RER values than the bench press $(p<0.001)$ (Table $1)$.

\section{UBIST Performance Post Exercise}

Repeated measures ANOVA did reveal a significant main effect for time $(\mathrm{F}=65.461, p<$ 0.001 ) for the upper body isometric test. However, the interaction of the exercise type and time was non-significant $(\mathrm{F}=0.585, p=0.742)$ (Figure 1).

\section{Salivary Testosterone and Cortisol Responses}

Analysis of testosterone concentrations via repeated measures ANOVA did not reveal a significant main effect for time $(\mathrm{F}=0.677, p=$ 0.416 ) nor significant interaction effect for the exercise type by time $(\mathrm{F}=0.904, p=0.411)$. Similarly, a repeated measures ANOVA examining cortisol did not reveal a significant main effect for time $(F=3.19, p=0.081)$ or interaction effect for the exercise type by time $(\mathrm{F}=$ 0.7699, $p=0.4684$ ). Additionally, when a testosterone to cortisol ratio was analyzed no significant main $(\mathrm{F}=0.038, p=0.846)$ or interaction effects $(\mathrm{F}=1.00, p=0.374)$ were found (Figures 2 and 3). 
Table 1

Heart Rate, Oxygen Consumption and Respiratory Exchange Ratio Values by Exercise Type $(M \pm S D)$

\begin{tabular}{llll}
\hline Exercise Condition & Average Heart Rate & $\begin{array}{l}\text { Average Oxygen } \\
\text { Consumption }(\mathrm{ml} \\
\left.\mathrm{O}_{2} / \mathrm{kg}^{*} \mathrm{~min}\right)\end{array}$ & Average RER \\
\hline Quad & $126.6 \pm 37.5$ & $13.49 \pm 2.58$ & $1.08 \pm 0.06 \#$ \\
Dual & $112.0 \pm 33.9$ & $12.73 \pm 1.83$ & $1.04 \pm 0.06 \#$ \\
Bench Press & $130.0 \pm 46.2$ & $11.25 \pm 2.69$ & $1.18 \pm 0.10$ \\
\hline
\end{tabular}

\# significantly different from bench press exercise condition $(p<0.05)$

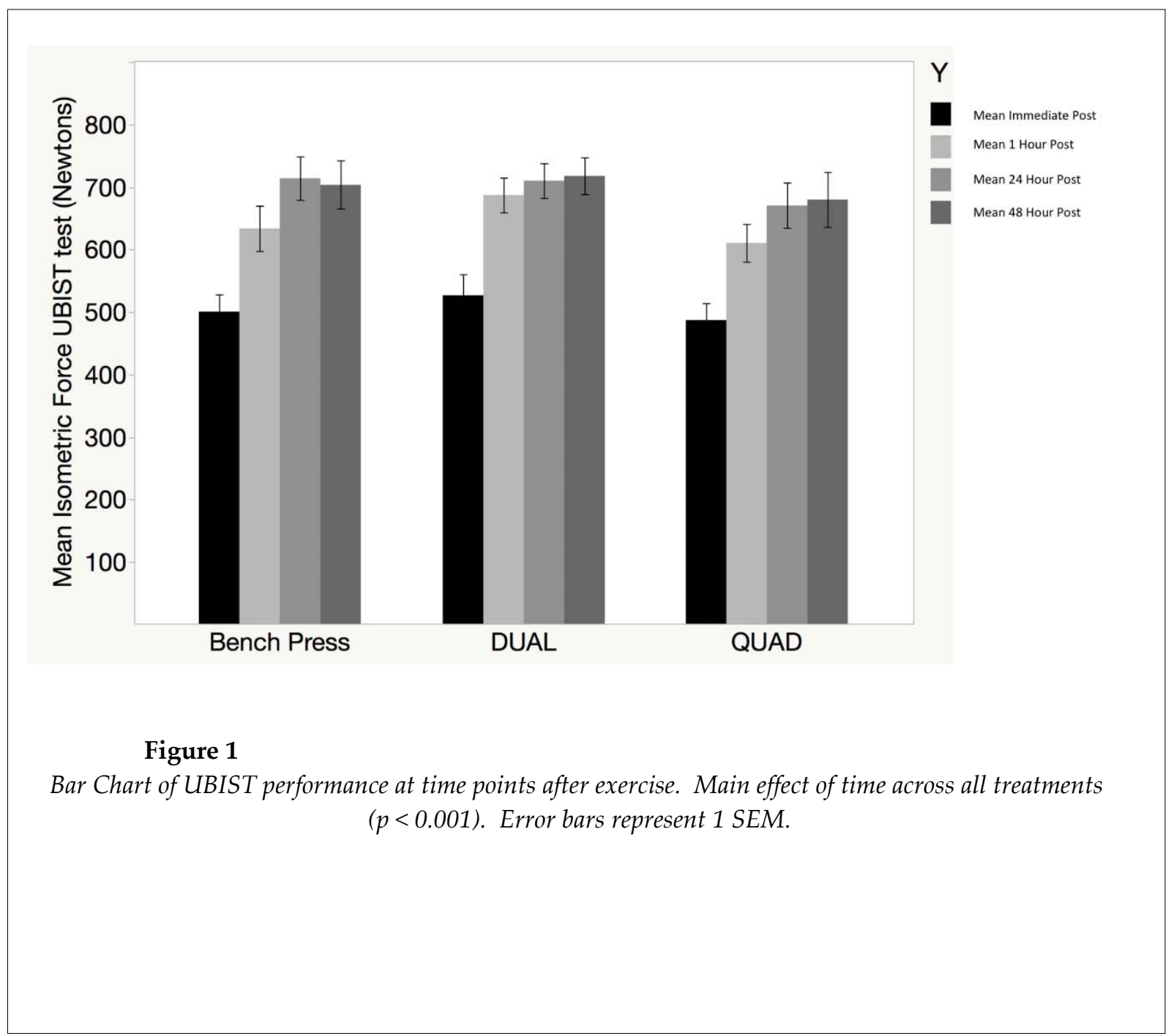




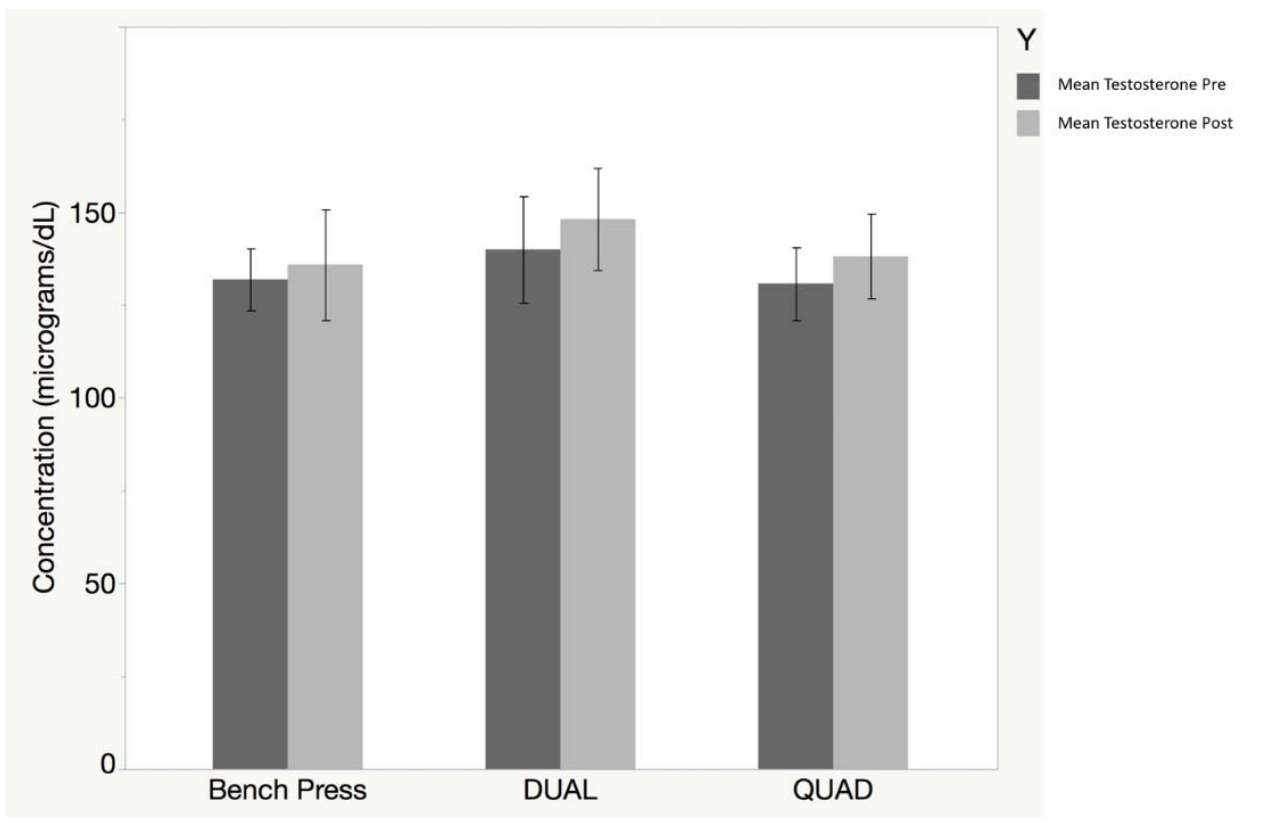

Figure 2

Pre and Post Exercise Testosterone concentrations by exercise conditions.

No significant main or interaction effects for time, treatment or treatment by time interaction $(p>0.05)$. Error bars represent 1 SEM.

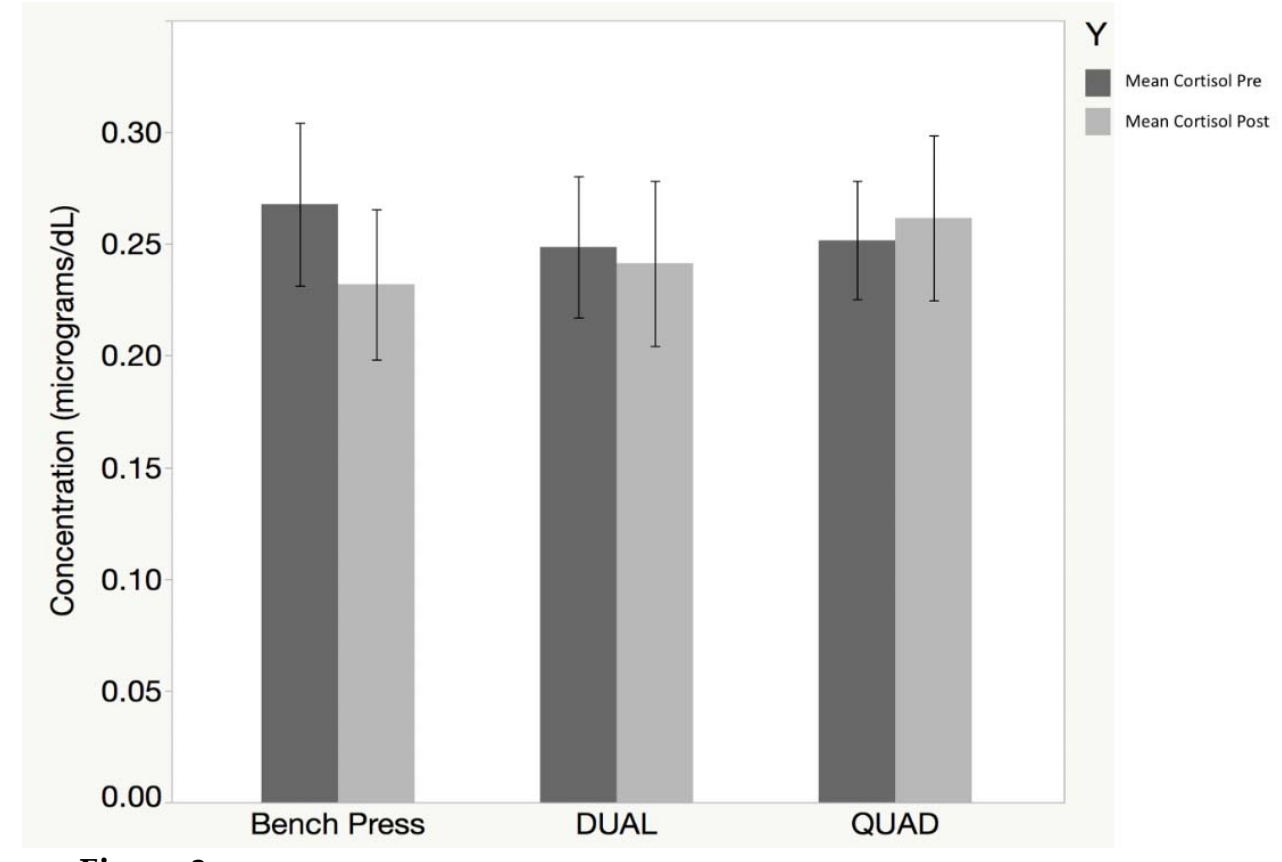

Figure 3

Pre and Post Exercise Cortisol concentrations by exercise conditions. No significant main or interaction effects for time, treatment or treatment by time interaction $(p>0.05)$. Error bars represent 1 SEM. 


\section{Discussion}

The present investigation expands on the data available in the literature about the effects of suspension exercise systems. The results of the study showed increases in oxygen consumption with a 4 strap push-up over a work matched bench press and additionally reductions in RER with both a 2 and 4 strap suspension push-up compared to the bench press. These findings are similar to those reported by Snarr et al. (2014) who suggested that the use of suspension exercise may result in a moderate aerobic training effect. The addition of the RER measurement in the present study may influence the interpretation of the oxygen consumption changes. Given that VO2 was elevated and the RER reduced in the QUAD condition as compared to the matched bench press, it is likely that additional supporting muscle groups are the source of the increase in the metabolism. If the prime movers in the pushup/bench press were stressed differently during the exercise, one would have expected to see an increase in oxygen consumption and increase in RER signifying greater carbon dioxide as a result of increased reliance on anaerobic pathways. Our data demonstrated that expired carbon dioxide was similar in all conditions. Based upon this, it may not be likely that the increased oxygen consumption would result in an aerobic training effect over time. Additionally, the QUAD treatment resulted in 3.8 METS versus the bench press, which elicited an average of 3.2 METS. While both of these qualify as moderate intensity exercise (Garber et al., 2011), the participants would have a difficult time performing a sufficient volume of training in this mode to meet the recommended $\geq 150 \mathrm{~min}^{*} \mathrm{wk}^{-1}$ for this to be used as a cardiovascular mode of training. Additionally, the increase of 0.6 METS is likely to be trivial in a long-term training plan.

The immediate reductions in isometric force production and subsequent recovery between conditions do offer some useful information about the impact of suspension exercise on the prime movers in the bench press/push-up movement. The mean force production was very similar immediately post exercise $(\mathrm{QUAD}=487.2 \mathrm{~N}, \mathrm{DUAL}=526.6 \mathrm{~N}$, bench press $=500.6 \mathrm{~N}$ ) and all conditions induced increases in isometric strength at approximately the same rate of $100 \mathrm{~N}$ after $1 \mathrm{hr}$ and $200 \mathrm{~N}$ over
$24 \mathrm{hr}$. This information may be valuable for strength and conditioning professionals in prescribing adequate rest between training as it appears the plateau in isometric force recovery occurs no later than 24 hours post exercise.

The information presented in this paper has utility for the coach/practitioner. According to an article published in the National Strength and Conditioning Journal (Berg, 1982), knowledge about the energy systems involved in training athletes is valuable to coaches. In addition to understanding energy system demands for the purposes of organizing training, it is also important to understand the demands of training methods in order to determine appropriate nutritional strategies for athletes (Morehen et al., 2016).

The limitations to the present study are somewhat inherent to the design of suspension exercise systems. These devices rely on gravitational forces based upon the mass of the user. The average support weight during the push-up exercise for the participants was $61.6 \pm$ $6.4 \mathrm{~kg}$ or $75 \%$ of body mass which is similar to other reports in the literature (Melrose and Dawes, 2015). As such many of the participants in the study would choose to perform bench press exercise with a higher load, which might augment endocrine responses (Gaviglio et al., 2015) as compared to what was seen in the present study. Therefore, the use of a work-matched protocol may not have reflected participants' normal training in the bench press exercise, and may not have elicited similar results to training in a more natural manner. Additionally, the use of recreationally trained individuals may limit the application of the findings to athletic populations.

\section{Conclusion}

Coaches and personal trainers should be aware that in comparable situations training with suspension systems and free-weights results in relatively similar acute responses. Though there was an increase in oxygen consumption that was statistically significant, the magnitude of the overall change may practically limit significance. Recovery of isometric strength was also comparable between conditions, but it does suggest that $24 \mathrm{hr}$ of recovery is necessary for a plateau in recovery of isometric strength to be seen. Strength and conditioning coaches and 
personal trainers can use this information to guide the selection of suspension exercise for athletes and recreational training based upon a desire to increase oxygen consumption. However, the study does not support the use of these devices to create a post exercise anabolic state determined through salivary testosterone and cortisol.

\section{Acknowledgements}

Partial results from this study have been presented at the National Strength and Conditioning Association Convention. TruFit, LLC provided financial support for this project.

\section{References}

Ahn RS, Lee YJ, Choi JY, Kwon HB, Chun SI. Salivary cortisol and DHEA levels in the Korean population: age-related differences, diurnal rhythm, and correlations with serum levels. Yonsei Med J, 2007; 48(3): 379-388

Beaven CM, Gill ND, Cook CJ. Salivary testosterone and cortisol responses in professional rugby players after four resistance exercise protocols. J Strength Cond Res, 2008; 22(2): 426-432

Bellar D, LeBlanc NR, Campbell B. The effect of 6 days of alpha gylcerylphosphorylcholine on isometric strength. J Int Soc Sports Nutr, 2015; 12: 42. Doi: 10.1186/s12970-015-0103-x

Bellar D, Marcus L, Judge LW. Validation and reliability of a novel test of upper body isometric strength. J Hum Kinet, 2015; 47: 189-195

Berg K. Anaerobic Conditioning: Training the three energy systems. NSCA Journal, 1982; Feb-Mar: 48-50

Borreani S, Calatayud J, Colado JC, Tella V, Moya-Nájera D, Martin F, Rogers ME. Shoulder muscle activation during stable and suspension push-ups at different heights in healthy subjects. Physical Therapy in Sport, 2015; 16(13): 248-254

Byrne JM, Bishop NS, Caines AM, Crane KA, Feaver AM, Pearcy GE. Effect of using a suspension training system on muscle activation during the performance of a front plank exercise. J Strength Cond Res, 2014; 28(11): 3049-3055

Calatayud J, Borreani S, Colado JC, Martin F, Rogers M. Muscle activation levels in upper-body push exercises with different loads and stability conditions. Phys Sportsmed, 2014; 42: 106-109

Calatayud J, Borreani S, Colado JC, Martin FF, Rogers ME, Behm DG, Anderen LL. Muscle activation during push-ups with different suspension training system. J Sports Sci Med, 2014; 13, 502-510

Dudgeon WD, Herron JM, Aartun JA, Thomas DD, Kelley EP, Scheett TP. Physiological and metabolic effects of a suspension training workout. International Journal of Sports Science, 2015; 5(2): 65-72

Freeman S, Karpowicz A, Gray J, McGill S. Quantifying muscle patterns and spine load during various forms of the push-up. Med Sci Sports Exerc, 2006; 38: 570-577

Gaviglio CM, Osbourne M, Kelly VG, Kilduff LP, Cook CJ. Salivary testosterone and cortisol responses to four different rugby training exercise protocols. Eur J Sport Sci, 2015; 15: 497-504

Garber CW, Blissmer B, Deschenes M, Franklin B, Lamonte M, Lee I-M, Nieman D, Swain D. Quantity and quality of exercise for developing and maintaining cardiorespiratory, muscloskeletal, and neuromotor fitness in apparently healthy adults: Guidance for prescribing exercise. Med Sci Sports Exerc, 2011; 43: 1334-1359

Gołaś A, Maszczyk A, Pietraszewski P, Stastny P, Tufano JJ, Zając A. Effects of pre-exhaustion on the patterns of muscular activity in the flat bench press. J Strength Cond Res. 2017; 31(7): 1919-1924

Klentrou P, Giannopoulou A, McKinlay BJ, Wallace P, Muir C, Falk B, Mack D. Salivary cortisol and testosterone response to plyometric exercise in 12- to 14- year-old boys. Appl Physiol Nutr Metab, 2016; 41(7): 714-718

Maszczyk A, Gołaś A, Czuba, M, Król H, Wilk M, Stastný P, Goodwin J, Kostrzewa M, Zając A. EMG Analysis and Modelling of Flat Bench Press Using Artificial Neural Networks. SAJRPER. 2016; 38(1): 91-103 
Melrose D, Dawes J. Resistance Characteristics of the TRX ${ }^{\mathrm{TM}}$ Suspension Training System at different angles and distances from the hanging point. J Athl Enhancement, 2015; 4: 1. doi: 10.4172/2324-9080.1000184

Morehen JC, Bradley WJ, Clarke J, Twist C, Hambly C, Speakman JR, Morton JP, Close GL. The assessment of total energy expenditure during a 14-day in-season period of professional rugby league players using the doubly labelled water method. Int J Sport Nutr Exerc Metab, 2016; 26(5): 464-472

Mok NW, Yeung EW, Cho JC, Hui SC, Liu KC, Pang CH. Core muscle activity during suspension exercise. J Sci Med Sport, 2015; 18, 189-194

Prokopy MP, Ingersoll CD, Nordenschild E, Katch FI, Gaesser GA, Weltman A. Closed-kinetic chain upperbody training improves throwing performance of NCAA Division 1 softball players. J Strength Cond Res, 2008; 22: 1790-1798

Saeterbakken AH, van den Tillaar R, Seiler S. Effect of core stability training on throwing velocity in female handball players. J Strength Cond Res, 2011; 25: 712-718

Shirtliff EA, Granger DA, Likos A. Gender differences in the validity of testosterone measured in saliva by immunoassay. Horm Behav, 2002; 42: 62-69

Snarr RL, Esco MR. Comparison of electromyographic activity when performing and inverted row with and without a suspension device. JEP online, e 2013; 16: 51-58

Snarr RL, Esco MR. Electromyograhic comparison of traditional and suspension push-ups. J Hum Kinet, 2013; 9: 75-83

Snarr RL, Esco MR. Electromyographical comparison of plank variations performed with and without instability devices. J Strength Cond Res, 2014; 28: 3298-3305

Snarr RL, Esco MR, Nickerson RL. Metabolic and cardiovascular demands of a high-intensity interval exercise bout utilizing a suspension device. J Sport Human Perf, 2014; 2: 1-8

Yu K-H, Suk M-H, Kang S-W, Shin Y-A. Effects of combined resistance exercise with TRX on physical fitness and competition times in Fin swimmers. International Journal of Sport Studies, 2015; 5: 508-515

\section{Corresponding author:}

\section{David Bellar, Ph.D.}

Director and Associate Professor-School of Kinesiology

University of Louisiana at Lafayette

225 Cajundome Blvd.

Lafayette, LA 70506

Fax: $337-482-6278$

Office: $337-482-6615$

E-mail: dmb1527@louisiana.edu 